\title{
Optimization of College English Multimedia Teaching by Data Mining
}

\section{Technology}

\author{
Weiliang Gao ${ }^{1, a}$ \\ ${ }^{1}$ Foreign Languages School of Changchun Normal University, Changchun Jilin 130032 \\ ${ }^{a}$ email,
}

\begin{abstract}
Keywords: College English; Multimedia Teaching; Data Mining
\end{abstract}
Abstract. Along with the progress of the society and the development of information technology, Multimedia application technology plays a more and more important role in production and life, and multimedia teaching has become an indispensable part of college English teaching. Then, how to conduct date mining on the text data, image data, video data and network data in multimedia teaching resources has become a hot topic. To do this work will greatly promote the advancement of multimedia technology in the teaching system, and greatly improve the level of teaching and learning.

\section{Introduction}

Multimedia database system used in Colleges and universities is composed of multimedia database and multimedia database management system. Media database system is a general designation of multimedia data, which is compose of text data, image data, audio data, video data, and web page data, and multimedia database management system is to achieve analysis, arrangement and classification on these data resources.

In recent years, data mining technology is widely used in enterprise management and decision making, such as business management, production control, market analysis, engineering design and scientific exploration, etc. However, it is still rarely applied in college English teaching. With the rapid development of electronic technology, multimedia teaching plays a more and more important role in college English teaching, especially English learning teaching, which is basically multimedia teaching. In addition, many English exams are conduct on computer network. For example, in a listening teaching system, there is much valuable information, which could reflect the potential effects of English listening teaching. Therefore, we can utilize data mining technology to dig out the Valuable knowledge from this information, to guide the English teaching.

The effectiveness and improvement of English multimedia teaching $t$ is an important research topic. People generally improve it through questionnaires or evaluation, but the effect is limited. This paper propose to utilize data mining technology in application of college English multimedia teaching, and provide some reference for research in this area.

\section{Application of Data Mining in English Multimedia Teaching}

Currently, in college English teaching, teachers have developed a good addition to using fixed software packages, and in addition to this, multimedia courseware has also become an important task for teaching staff. Most of the multimedia courseware production is PowerPoint slideshow, or specialized educational software for making slide. Such teaching software is made based on 
teachers' own experience and teaching syllabus, and then it is constantly being improved according to student's study situation and the test situation.

In the actual teaching process, some teaching software could continuously feedback a large amount of data, such as listening courseware software, which can record the student's practice data in classroom. We can utilize data mining technology to process these data, to assess the extent of students to master course content and their learning effects, and then improve the courseware. Then, How to achieve this process? That is right what we are going to discuss as follow.

Data mining is a multi-stage process, including three main stages: data preparation, data mining, and results evaluation. The whole process is a constant cycle and repeated process, as shown in Fig.1.

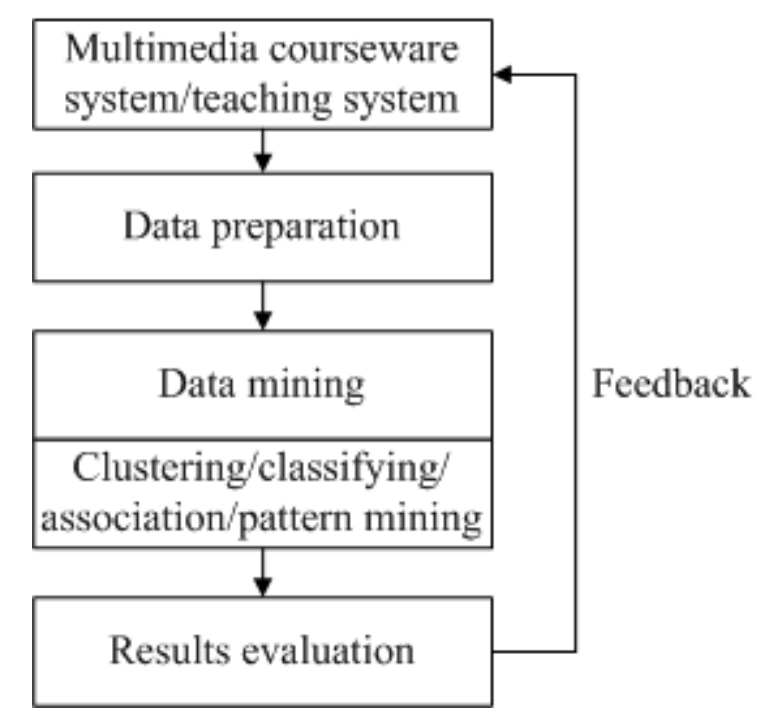

Fig. 1. Data mining for multimedia teaching

The main purpose of the data preparation is to extract the relevant data from the database according to the user's requirements, and ensure the integrity and consistency of the data. Data mining phase mainly includes: determining the purpose of data mining, using the correct and efficient algorithm to extract the knowledge from the database. While results evaluation mainly refers to the interpretation of the knowledge mining and presenting to users in an accessible way. Meanwhile, we may also need to check the consistency of knowledge in order to eliminate conflicting knowledge.

Data mining is a professional technology. In order to promote the application of data mining technology in college English teaching and bring convenience to the teaching staff, we developed the corresponding software system, which can not only be used as an additional system of multimedia teaching system, but also run as an independent system.

Data preparation. Data preparation is the data collection process for data mining, including active data collection and passive data collection. The former is obtained by means of a questionnaire or a direct test, while the latter is to convert the data in the classroom into required data. Passive data collection is done automatically, so Teachers avoid the initiative to get data to pay a lot of labor, only needs increase the conversion process in the original teaching system.

Classroom test is one of the main accesses to data. Since a number of multimedia teaching software is equipped with a number of corresponding classroom contact and test exercises, teacher can know about the student's learning situation through the test, and the data also reflects the potential link between teaching and student learning. 
The data object we set here includes professional, questions, answers, knowledge points and repeat times, etc. We design a special database for data collection and data mining.

Data mining. Data mining procedures require teachers to work with specialized technical personnel to develop. The data mining platform is designed to meet the functional requirements in college English multimedia teaching, and provide the interface that is simple and easy to use.

Depending on the type of mining knowledge, it is usually divided into clustering rule mining, classification rule mining, association rule mining and pattern mining, etc.

(1) Clustering rule mining

Clustering is to divide the data into different clusters, to make the distance between the individuals of the same cluster as small as possible, and meanwhile the distance between the individuals of the different cluster as large as possible. Although to represent the entire data set with a small number of clusters would lose some of the details of the data set, it effectively simplifies the representation of the data set.

Clustering analysis is an important human behavior. Early in childhood, through continuous improvement subconscious clustering model, human would learn how to distinguish between animals and plants. At present, clustering analysis has been applied in many aspects, including pattern recognition, data compression, image processing, sound processing, and market analysis, etc. By clustering analysis, people are able to identify dense and sparse areas, and thus find out the global distribution model and the interesting relationships between data properties.

Therefore, we can discover the group effect brought by the multimedia teaching through the clustering rule mining. Listening is one of the main aspects of learning English, and listening test data is the most easy to obtain. By clustering teachers can explorer how the questions affect the students. In addition, we can adopt vector space model clustering, and set questions, answers, accuracy and knowledge points as the clustering data.

(2) Classification rule mining

The role of classification is to divide an uncertain object to a known class. Generally, through the characteristics of known training data and classification results, find out reasonable description or model for each class, and then use these descriptions or models to classify the known new data set. People usually use production rules to represent the data mining results.

Teachers can explorer students' learning situation in terms of words, phrases and clauses, etc. We can test data as training data to identify common characteristics of the data.

(3) Association rule mining

The purpose of association rule mining is to dig out the hidden relationship between the data.

Assume $I=\{i 1, i 2, \ldots, i n\}$ as a collection of different items of $m$, given a transaction database $D$, each transaction $T$ is a subset of the items $T I$; each transaction has a unique identifier representation $T I D$, then we can think that transaction $T$ contains items set $X$. if $X T$, an association rule is an implication like $X==>Y$, then $X$ is called the premise of rule and $Y$ is call the result of rule. If there are $\mathrm{s} \%$ of transactions in transaction database contain $X Y$, then, we can deduce the association rule $X==>Y$ has certain support degree in $D$; if there are also c\% of transactions in transaction database contain $Y$ at the same time, then we can deduce the association rule $X==>Y$ has certain confidence level in $D$.

Therefore, using the association to discover the comprehension of sentences in the English learning teaching, e.g. we test a number of key words $X$ in a sentence, bringing about another meaning $Y$, and it is expressed by a rule $X==>Y$.

(4) Pattern mining 
The purpose of pattern mining is to dig out the characteristics between the data. Applying pattern mining in multimedia teaching, the teachers can find some common underlying patterns in student learning, such as patterns between words and phrases learning. Similarly, in pattern mining, the support and confidence degree also requires to be calculated.

Result evaluation. The result of data mining aims to provide valuable knowledge and rules for decision makers. The results of data mining may produce many rules and knowledge, but they may not be easy to be understood by teachers, so the interpretation of the output results can be used in language or graphics. According to the results of the mining, the teachers can evaluate the effect of the original teaching system or the teaching courseware, and thus improve the teaching methods and course content.

\section{Empirical Analysis}

After a period of English multimedia teaching, we chose a class of students as study object, and carry out empirical analysis on vocabulary learning feature clustering. We design A set of test papers, which involve about 600 vocabularies, and then we input the results of the test to the computer and carry out Numerical clustering analysis. At last, we count the results of the experiment, as shown in Tab.1.

Tab.1 Results of the experiment

\begin{tabular}{ccc}
\hline & Correct choice & Wrong choice \\
\hline noun & $87.6 \%$ & $89.5 \%$ \\
verb & $78.7 \%$ & $72.4 \%$ \\
adjective & $66.4 \%$ & $77.8 \%$ \\
\hline
\end{tabular}

We adopt center clustering method, and the data format is: question number, correct answer, wrong answer and choice results, etc. We can see from the testing results that, students in the same class have something common in vocabulary learning. Therefore, for the vocabularies that most students cannot grasp, teachers have better focus on teaching and practice.

Overall speaking, the data experiment shows that the data mining has a certain effect on the improvement of college English multimedia teaching.

\section{Conclusion}

In this paper, we give a theoretical approach to the process of using data mining techniques to optimize the multimedia teaching strategies of college English, and it is proved to be of great significance in improvement of college English multimedia teaching.

\section{References}

[1] Yang N Y, Center I T. Data Mining and Analysis for the Personalized Teaching of Multimedia Technology Course[J]. Computer Knowledge \& Technology, 2016.

[2] Jian-Min X U, Xiong J Z, Xiao J, et al. Development of "Data Mining" multimedia courseware based on undergraduate teaching of local colleges[J]. Electronic Design Engineering, 2012.

[3] Huang W C, Xiong L Y, Zeng H. The researching and realizing of multimedia teaching effect based on data mining[J]. Manufacturing Automation, 2010. 
[4] Liu F B. Improving College English Teaching by Using Data Mining[J]. Computer-Assisted Foreign Language Education, 2006.

[5] Zhimei Z. Application of data mining technology in the information technology of college english teaching[J]. Advance Journal of Food Science \& Technology, 2013, 5(7).

[6] Jiang F. Classification Rule Constructing based on Data Mining to Promote English Teaching[J]. Advances in Information Sciences \& Service Sciences, 2013, 5:1097-1104. 